medical records. All infants underwent postnatal cardiac ECHO, electroencephalogram and brain MRI, along with calculation of Bicetre severity score at admission. Embolization procedures were analysed based on timing and presence of peri- and post-procedural complications. Long-term survivors had standard neurocognitive assessment.

For the purpose of outcome analysis we divided patients into two period cohorts: 2001-2010 and 2011-2020.

Overall fourteen out-born neonates were diagnosed with chorioidal VGAM. Bicetre neonatal score were similar in both chronological sub-groups. First cohort comprised six neonates. Prenatal diagnosis of VGAM was obtained in one patient. Four newborns died owing to refractory heart failure without neuroembolization procedure. Two survivors underwent the intervention at fourth and eleventh day, respectively. Post-procedural analysis revealed minor intraventricular bleeding and neonatal seizures in one patient that eventually has been lost to follow up. Other survivor developed ischaemic lesions and hydrocephalus. Ventriculo-peritoneal drainage was performed.

Severe neurodevelopmental delay and visual impairment were observed on follow-up. Second cohort comprised eight neonates, all prenatally diagnosed. Three patients died: at the age of five (intra-procedural rupture of aneurism), fifteen (multi-organ failure), and 205 days (pulmonary hypertension and congestive heart failure). All infants undergone the first embolization within the first ten days of life. Satisfactory immediate outcome including control of cardiac failure was achieved in six patients. Post-procedural analysis revealed minor intraventricular bleeding $(n=2)$, ventriculomegaly $(n=2)$, presence of ischaemic lesions $(n=2)$, neonatal seizures $(n=2)$ and abnormal encephalogram $(n=2)$. Normal neurodevelopment was observed in four, and mild neuro-developmental delay was observed in one patient on follow-up.

Given a small sample size it is difficult to quantify the difference between the two groups in a statistically relevant manner. However, the observed difference in survival and longterm outcome between the cohorts are most probably related to an overall improvement of prenatal diagnosis, intensive care management and embolization techniques. Early neuroembolization may decrease the risk of refractory cardiac failure and improve the long-term neurocognitive outcome.

\section{IS THERE A GOOD CORRELATION OF MAGNETIC RESONANCE IMAGING AND CRANIAL ULTRASOUND IN BRAIN IMAGING IN PRETERM INFANTS AT TERM AGE?}

Benjak Vesna*, Ninković Dorotea, Ćaleta Tomislav, Vukšić Iva, Dasović Buljević Andrea, Bunoza Branka, Grðan Petra, Radoš Milan, Grizelj Ruža. Clinical hospital centre Zagreb

\subsection{6/archdischild-2021-europaediatrics.125}

The aim of this study was to compare MRI findings in preterm infants at term age with cranial ultrasounds as predictors in the assessment of neurodevelopment outcomes and need for early habilitation procedures.

The study included 64 premature infants gestational age under 32 weeks who were admitted in NICU at University Hospital Centre Zagreb between years 2013. and 2016. and underwent brain MRI examination. 53,12\% examinees were boys and 48,8\% were girls. Mean gestational age was 29,0 $(+/-3,2)$ weeks, and mean birth weight was $1336(+/-466) \mathrm{g}$. Reanimation at birth was necessary in $50 \%$ of examinees. Some method of mechanical ventilation (invasive or noninvasive) was used in $70 \%$ of examinees, in duration of approximately 22 (1-150) days. Cranial ultrasound scans were performed at te age of one day, four days and seven days and then once a week and at term age. Brain MRI examinations were performed at gestational age of 32 weeks and at term age. A statistical analysis of the correlation of ultrasound findings and brain MRI examinations at term age was performed. Cranial ultrasound findings were classified into four group by presence of IVH or leukomalatia: (0-normal finding; 1-mild hyperechogenicity or IVH grade I/II; 2-IVH with ventricular dilatation or presence of periventricular cysts; 3-ventriculomegaly or hydrocephalus and severe periventricular leukomalatia) and in two groups by presence of talamic lesions (present or not present).

MRI findings were classified as normal, mildly abnormal (mild gliosis); moderately abnormal (ventricular dilatation with gliosis, talamic lesions) and severely abnormal group (severe gliosis/PVL and hydrocephalus).

Group of examinees with Apgar score $<5$ after 1st minute had moderately to severly abnormal MRI findings. Mechanically ventilated premature infants showed statistically significantly higher $(\mathrm{p}<0,001)$ correlation with severe MRI abnormalities.

Comparation of ultrasound and MRI findings didn't show good correlation (Kendall Tau $=0,715 ; \mathrm{p}<0,05$ ). Hi-square test showed ultrasound scans statistically significantly overestimate the number of premature infants with lesions (presence of IVH or leukomalatia). The number of talamic lesion was also overestimated by ultrasound scans (Hi-square test 8,824; $\mathrm{p}=0,004)$.

But, correlation between methods considering moderately to severely abnormal MRI findings was better (Hi-square test $13,53 ; \mathrm{p}=0,003)$.

Magnetic resonance imaging is superior method comparing to brain ultrasound, but despite that brain ultrasound is still unavoidable as standardized routine screening method. Together they form the gold standard in the assessment of neurodevelopment outcomes and need for early habilitation procedures in premature infants.

\begin{tabular}{l}
\hline 126 \\
A BROKEN NEC: CASE STUDY OF IDIOPATHIC SUPERIOR \\
MESENTERIC VEIN THROMBOSIS IN A PREMATURE \\
NEONATE
\end{tabular}

Cormac Duff*, Niazy Al Assaf. University Maternity Hospital, Limerick

\subsection{6/archdischild-2021-europaediatrics. 126}

Introduction Neonates are the pediatric population at highest risk for development of venous thromboembolism. ${ }^{1}$ Intravascular catheters are a major risk factor.

Other risks include prematurity, neonatal asphyxia and sepsis. $^{2}$ Neonatal superior mesenteric venous thrombosis has not previously been described in medical literature.

Case A preterm female infant was born by emergency Caesarean section at 28 weeks' gestational age, weighing $760 \mathrm{~g}$. The indication was severe symmetrical intrauterine growth restriction and absent end diastolic flow.

Her mother was an Irish 23-year-old primigravida, with a background of severe depression and smoking. The neonate required continuous positive airway pressure (CPAP) and artificial surfactant. She required an umbilical venous catheter 